\title{
Evidence-informed physical activity guidelines for people with spinal cord injury
}

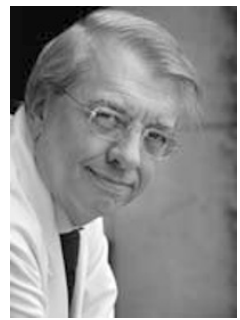

JJ Wyndaele, Editor

Antwerp University Hospital, Antwerp, Belgium

E-mail: spinalcord@uza.be

Dear Spinal Cord reader,

In this issue there is a large contribution from Martin Ginnis and Hicks, who develop exercise guidelines to improve physical capacity and muscular strength in individuals with spinal cord lesion. The evidence base for the guideline development process consisted of a systematic review and quality appraisal of research examining the effects of exercise on physical fitness among people with SCI. A multidisciplinary expert panel deliberated the evidence and generated the guidelines. Pilot testing led to refinement of the wording and presentation of the guidelines. The expert panel concluded that for important fitness benefits, adults with a SCI should engage in at least 20 min of moderate to vigorous intensity aerobic activity 2 times per week and strength training exercises 2 times per week, consisting of 3 sets of 8-10 repetitions of each exercise for each major muscle group.

A second literature review evaluated the effects of an exercise intervention on at least one of four main components of physical fitness: physical capacity, muscular strength, body composition and functional performance. It included studies reporting at least one of the following outcomes: oxygen uptake/consumption, power output, peak work capacity, muscle strength, body composition, exercise performance, or functional performance. Though most studies were of low quality, the evidence was consistent that exercise is effective for improving aspects of fitness. There is strong evidence that exercise, performed 2-3 times per week at moderate-to-vigorous intensity, increases physical capacity and muscular strength in the chronic SCI population; the evidence is not strong with respect to effects of exercise on body composition or functional performance. There were insufficient high quality studies in the acute SCI population to draw any conclusions.

There have been several previous publications on exercise in Spinal Cord which you may find of interest (Spinal Cord 2008; 46: 216-221; Spinal Cord 2010; 48: 355, 388-392, 393-399).

There are several other interesting manuscripts in this issue. In animal research, Klironomos et al. developed a model in the rabbit of cervical spondylotic myelopathy (CSM) which reproduces the temporal evolution of the disease and creates a local microenvironment at the site of spinal cord compression which shares the same characteristics as in humans.

Imajo et al. evaluated relative vulnerability of various spinal tracts in C3-4CSM with multimodal spinal cord evoked potentials. The study showed the lateral parts of the posterior funiculus mediating upper limb sensory function were more vulnerable than the lateral corticospinal tract. It confirmed that the lateral parts of the posterior funiculus were most vulnerable in three spinal tracts. Finger numbness in mild, early stage CSM was associated with this pathology.

Kawu et al. determined the cost of acute phase of injury among spinal cord injured patients managed conservatively in Nigeria. Such studies are important to help plan care on different levels and help top control and limit the financial burden for patients and relatives.

Engkasan Julia et al. demonstrated that abdominal binders can be used as an effective method to improve cough ability in spinal cord injured patients, with triple-strap abdominal binder achieving greater cough peak expiratory flows.

Backhaus et al. want to increase awareness that pressure sores significantly increase the risk of developing Fournier's gangrene in patients with SCI.

Enjoy reading.

Spinal Cord (2011) 49, 1087; doi:10.1038/sc.2011.122 